\title{
MIGRAÇÃO VENEZUELANA: REFLEXÕES SOBRE COMUNICAÇÃO VERBAL PRODUZIDA POR ENFERMEIROS DA ATENÇÃO PRIMÁRIA À SAÚDE
}

\author{
VENEZUELAN MIGRATION: REFLECTIONS \\ ON VERBAL COMMUNICATION PRODUCED BY \\ PRIMARY HEALTH CARE NURSES
}

\section{MIGRACIÓN VENEZOLANA: REFLEXIONES SOBRE LA COMUNICACIÓN VERBAL PRODUCIDAS POR ENFERMERAS DE ATENCIÓN PRIMARIA DE SALUD}

\author{
Paulo Sérgio da Silva ${ }^{1}$
}

Como citar este artigo: Silva PS. Migração venezuelana: reflexões sobre comunicação verbal produzida por enfermeiros da Atenção Primária à Saúde. Rev baiana enferm. 2021;35:e45296.

Objetivo: refletir sobre a comunicação verbal realizada por enfermeiros da Atenção Primária à Saúde no cuidado dirigido ao migrante venezuelano. Método: trata-se de estudo descritivo de caráter qualitativo. Pesquisa realizada com dez enfermeiros distribuídos em nove unidades básicas de saúde da família no município de Boa Vista, Roraima. A técnica de coleta de dados foi a entrevista semiestruturada. Os dados brutos foram gravados e seus conteúdos transcritos para análise de conteúdo. Resultados: as unidades de registros foram organizadas na categoria analítica intitulada: "O idioma como indutor de comunicação verbal prejudicada no cuidado realizado por enfermeiros ao migrante venezuelano na atenção primária à saúde". Considerações finais: as reflexões sobre a comunicação verbal realizada por enfermeiros da atenção primária à saúde no cuidado dirigido ao migrante venezuelano apontaram o idioma como uma barreira de comunicação. O falar pausadamente, a repetição de mensagens e o esforço para compreensão da língua espanhola foram estratégias adotadas.

Descritores: Comunicação. Barreiras de Comunicação. Enfermeiras e Enfermeiros. Atenção Primária à Saúde. Migração Humana.

Objective: to reflect on the verbal communication performed by primary bealth care nurses in the care directed to Venezuelan migrants. Method: this is a descriptive study of qualitative character. A study conducted with ten nurses distributed in nine basic family health units in the municipality of Boa Vista, Roraima. The data collection technique was the semi-structured interview. The raw data were recorded and their contents transcribed for content analysis. Results: the units of records were organized in the analytical category entitled: "The language as an inducer of verbal communication impaired in the care performed by nurses to Venezuelan migrants in primary health care." Final considerations: the reflections on verbal communication performed by primary bealth care nurses in the care directed to Venezuelan migrants pointed to the language as a communication barrier. Speaking slowly, the repetition of messages and the effort to understand the Spanish language were strategies adopted.

Descriptors: Communication. Communication Barriers. Nurses. Primary Health Care. Human Migration.

Enfermeiro. Doutor em Ciências. Professor Adjunto da Universidade Federal de Roraima. Boa Vista, Roraima, Brasil. pssilva2008@gmail.com. http://orcid.org/00000003-2746-2531. 
Objetivo: reflexionar sobre la comunicación verbal realizada por las enfermeras de atención primaria de salud en la atención dirigida a migrantes venezolanos. Método: se trata de un estudio descriptivo de carácter cualitativo. Estudio realizado con diez enfermeras distribuidas en nueve unidades básicas de salud familiar en el municipio de Boa Vista, Roraima. La técnica de recogida de datos fue la entrevista semiestructurada. Los datos en bruto fueron registrados y su contenido transcrito para el análisis de contenido. Resultados: las unidades de registros se organizaron en la categoría analítica titulada: "El lenguaje como inductor de la comunicación verbal deteriorada en la atención que realizan las enfermeras a los migrantes venezolanos en atención primaria de salud”. Consideraciones finales: las reflexiones sobre la comunicación verbal realizadas por las enfermeras de atención primaria de salud en la atención dirigida a los migrantes venezolanos apuntaron al idioma como barrera de comunicación. Hablando pausadamente, la repetición de mensajes y el esfuerzo por entender el idioma español fueron estrategias adoptadas.

Descriptores: Comunicación. Barreras de Comunicación. Enfermeras y Enfermeros. Atención Primaria de Salud. Migración Humana.

\section{Introdução}

O projeto investigativo intitulado "Rastreamento de Saberes e Práticas Assistenciais, Gerenciais e Educacionais no Âmbito da Atenção Primária à Saúde (APS)", cadastrado no Departamento de Pesquisa da Universidade Federal de Roraima (UFRR), tem sido orientador de profícuos diagnósticos científicos sobre o saber-fazer-gerir-educar em saúde no extremo Norte do Brasil. Esta região, nos últimos anos, tem sido impactada pelas migrações.

Certamente, o debate em torno desse tema-problema coloca em relevo a migração como um fenômeno nada recente e de amplitude global. Isso porque pouco mais ou menos de um sétimo da população mundial vive fora do seu local de origem ${ }^{(1)}$. Nesse contexto, estima-se que o atual cenário humanitário venezuelano sem precedentes já forçou 2 a 3 milhões de pessoas a fugirem do país. A maioria dos migrantes venezuelanos que adentram o Brasil à procura de condições adequadas de vida segue diferentes rotas migratórias na região amazônica, a saber: permanecer no próprio estado de Roraima; encaminhar-se para o estado do Amazonas - em destaque, para a capital Manaus; ou buscar interiorização, pela via aérea, para o restante dos estados brasileiros $^{(2)}$.

Especificamente, as cidades do estado de Roraima, destino desses migrantes venezuelanos, em sua grande maioria, não possuem estrutura apta para recebê-los. Os principais municípios escolhidos por esses migrantes são Pacaraima e Boa Vista, que possuem aproximadamente
16 mil e 300 mil habitantes, respectivamente. Portanto, apresentam dificuldades para recepcionar adequadamente mais de 30 mil migrantes venezuelanos, em especial no que se refere ao atendimento em educação e saúde, principais setores afetados ${ }^{(3)}$.

Quando se pensa na produção de cuidado em saúde, é preciso situar o migrante venezuelano no encontro com o enfermeiro nos serviços que compõem a Rede Básica (RB), compreendida como "[...] uma aposta de fazer chegar o cuidado em saúde aos diferentes territórios em que a vida é produzida. Cuidado contextualizado, que reconhece a singularidade da produção de cada existência e também as circunstâncias específicas da vida em cada território, em função de relações que ampliam ou constrangem a potência do viver" ${ }^{(4: 71)}$.

Fala-se de singularidades entendidas como objetos práticos dos enfermeiros que operam modos inclusivos de gerir a produção do cuidado na APS ou mesmo nos territórios onde a vida do migrante venezuelano ganha sentidos existenciais. A incompreensão desse migrante sobre a política de saúde brasileira, a sobrecarga das equipes básicas de saúde, reflexo da elevada demanda por assistência básica, e a superlotação dos espaços físicos são desafios cotidianamente vivenciados por enfermeiros nas Unidades Básicas de Saúde (UBS) da cidade de Boa Vista. Tudo isso atesta que a migração venezuelana é um fenômeno prioritário no campo da saúde pública ${ }^{(5)}$. 
Os problemas relacionados às diferenças culturais influenciam diretamente o saber-fazer saúde, no contexto da APS, junto aos migrantes venezuelanos. Nessa perspectiva, condições sociais e trabalhistas, costumes e crenças em saúde, estigma e discriminação, dificuldade para acessar os serviços de saúde, o próprio processo de comunicação estabelecido entre o enfermeiro e a pessoa migrante funcionam como determinantes sociais da saúde migratória ${ }^{(6)}$.

Aqui, a comunicação é compreendida como um compartilhamento de mensagens enviadas e recebidas que são capazes de exercer influências comportamentais nas pessoas envolvidas no cuidado. Cabe complementar que as mensagens presentes na comunicação podem ser transmitidas de maneira verbal ou não verbal e situam-se no ambiente em que ocorre a interação entre o emissor e o receptor do conteúdo ${ }^{(7)}$. Desse modo, o enfermeiro deve criar estratégias para superar "a distância" nesse ambiente, tornando, assim, a assistência efetiva do ponto de vista comunicacional ${ }^{(8)}$.

Sobre isso, uma base reflexiva referente à comunicação verbal estabelecida entre enfermeiros da APS e os migrantes venezuelanos traz à tona conhecimentos sobre a produção de cuidado com base em diálogos terapêuticos envolvendo idiomas distintos. Sendo assim, a comunicação verbal, ao utilizar as palavras expressas por meio da fala ou da linguagem escrita, é o instrumento básico do cuidar fundamental, para que o enfermeiro conheça e aplique, em sua prática clínica, a nomenclatura diagnóstica de enfermagem adequada às situações em que há prejuízo da comunicação verbal $^{(9)}$.

Baseado nessas afirmativas, o presente estudo abre possibilidades para a produção de reflexões sobre subjetividades que ocorrem pelo encontro dos idiomas português-espanhol em um espaço comum de cuidar. O lugar desses conteúdos revela um conjunto de conhecimentos, habilidades e atitudes relacionais apreendidas e protagonizadas por enfermeiros da APS, que ganham novos contornos quando a cultura do povo venezuelano é considerada.

Com esses fios introdutórios, emerge a seguinte questão norteadora desta investigação:
Como a comunicação verbal é percebida por enfermeiros da APS nos encontros de cuidado com os migrantes venezuelanos? Suscitar outras indagações implica na compreensão da complexidade envolvida no processo comunicacional verbal em um plano intercultural, sobretudo quando se considera a relação dos idiomas português-espanhol como uma lacuna do conhecimento a ser investigada no domínio do cuidado de enfermagem produzido na APS. Dessa forma, o objetivo do estudo foi refletir sobre a comunicação verbal realizada por enfermeiros da APS no cuidado dirigido ao migrante venezuelano.

\section{Método}

Trata-se de um estudo descritivo com abordagem qualitativa. Este tipo de investigação possibilita, ao pesquisador, responder questões mais particulares, em que a verdade da experiência e da vivência é orientada coletivamente para o que é correto, plausível e prático. A opção pela seleção desse tipo de abordagem metodológica deve-se à sua vitalidade no campo da saúde e à proposição de respostas epistemológicas inovadoras no campo social e da saúde ${ }^{(10)}$. Toda a pesquisa foi orientada pelos critérios listados no guia COREQ.

O grupo social envolvido neste estudo foi representado por dez enfermeiros atuantes na APS em Boa Vista (RR). A seleção obedeceu aos seguintes critérios de inclusão: tempo de atuação na UBS igual ou superior a três meses, ter realizado cinquenta ou mais atendimentos em saúde junto à população migrante venezuelana. Foram excluídos deste estudo: enfermeiros estrangeiros, enfermeiros licenciados ou afastados de suas atividades laborais e professores-enfermeiros vinculados às instituições de ensino superior que acompanhavam acadêmicos de enfermagem em suas atividades práticas de estágio supervisionado obrigatório.

O estudo foi realizado em Boa Vista, capital de Roraima, estado localizado na Amazônia Legal. Boa Vista é uma cidade em pleno desenvolvimento, que possui indicadores elevados de desenvolvimento humano entre o Norte e o Nordeste do Brasil. O local escolhido para realização 
do estudo foi a Rede de Atenção Psicossocial (RAPS) do município de Boa Vista (RR), que se divide em 8 macroáreas com 34 UBS.

A inclusão dos cenários do estudo foi realizada por conveniência e orientada pela proximidade dos abrigos destinados ao acolhimento de refugiados e migrantes venezuelanos em Roraima. Foram excluídas deste estudo as UBS destinadas exclusivamente ao atendimento do Coronavírus.

Inicialmente, a proposta investigativa, a carta de anuência e o memorando de aprovação do Comitê de Ética em Pesquisa (CEP) da UFRR foram apresentados aos responsáveis técnicos pelas UBS de Boa Vista. Em seguida, os enfermeiros foram convidados para participar do estudo, mediante ligações telefônicas e mensagens via aplicativo WhatsApp. No ato do convite, eles foram informados quanto aos objetivos da pesquisa, procedimentos metodológicos de construção dos dados e a necessidade de cumprimento das medidas de biossegurança para prevenção da Doença do Coronavírus. Após aceite, foi agendado individualmente o melhor horário e local para a realização do encontro investigativo.

A construção dos dados foi orientada por um roteiro de entrevista semiestruturado, contendo três questões que abordavam os seguintes temas: práticas de cuidar realizadas pelo enfermeiro na APS, comunicação verbal e não verbal no contexto migratório venezuelano. Todas as entrevistas foram obtidas com auxílio de um gravador de voz portátil e salvas em formato de áudio MP3 para posterior transcrição e análise.

Todo o material produzido foi transcrito e analisado segundo o referencial teórico-analítico de conteúdo disposto em Bardin. Esta técnica de análise textual caracteriza-se por procedimentos sistemáticos e objetivos de descrição de conteúdos presentes nas mensagens ${ }^{(11)}$. As unidades de registro advindas do processo analítico foram organizadas na categoria intitulada: "O idioma como indutor de comunicação verbal prejudicada no cuidado realizado por enfermeiros com o migrante venezuelano na APS".
O estudo obedeceu às diretrizes previstas na Resolução n ${ }^{\circ} 466 / 2012$ do Conselho Nacional de Saúde. O projeto de pesquisa foi submetido ao CEP da UFRR e aprovado pelo Parecer $n^{\circ}$ 4.701.055 emitido no ano de 2021. Toda a produção dos dados foi precedida pela leitura e assinatura do Termo de Consentimento Livre e Esclarecido (TCLE) e do Termo de Autorização de Gravação de Voz (TAGV). O anonimato dos participantes foi garantido pelo uso da palavra identificadora Participante, seguida de um número ordinal sequencial crescente, correspondente à ordem de realização da entrevista.

\section{Resultados}

Os conteúdos que versam sobre a diferença de idiomas, presentes no encontro estabelecido entre o enfermeiro e o migrante venezuelano no contexto da APS, induzem à produção de reflexões que colocam em destaque a comunicação verbal prejudicada envolvida no cuidado de enfermagem. Isso permitiu a inferência do idioma como uma barreira linguística envolvida na produção do cuidado protagonizado pelo enfermeiro ao migrante venezuelano no contexto da APS. Esses desafios de natureza linguística foram identificados em unidades de registros que podem ser visualizados nos depoimentos a seguir:

Falta de entendimento. É explicado o atendimento, e os venezuelanos apresentam dificuldade para compreender. (Participante 1).

O idioma dificulta muito. Eles [migrantes venezuelanos] falam muito rápido. Eu tenho que parar e pedir para falarem mais devagar. (Participante 2).

É muito difícil que eu entenda o que eles [migrantes venezuelanos] estão falando [...] muitas vezes, morremos de explicar, e eles não conseguem entender. (Participante 3).

Uma das barreiras que pega mesmo é a questão linguística. Não que não dê pra fazer o trabalho. Conseguimos, mas dificulta. (Participante 4).

Temos que esperar que eles [migrantes venezuelanos] se acalmem, falem mais devagar, para podermos compreender o idioma. (Participante 5).

O idioma. Temos que ficar tentando entender o espanhol. (Participante 6).

A lingua limita a assistência, deixando a gente impotente na resolutividade dos problemas. (Participante 7). 
Os imigrantes venezuelanos não entendem por completo as orientações fornecidas em português. (Participante 8).

A barreira linguística torna o atendimento demorado. Tenbo que ficar repetindo, para me certificar de que a informação foi assimilada. (Participante 9).

Alguns venezuelanos não entendem quase nada da nossa lingua; isso atrapalha muito a assistência de enfermagem. (Participante 10).

O desafio de melhorar a qualidade dos cuidados realizados por enfermeiros da APS perpassa pela comunicação verbal. Nela as orientações, explicações, emoções, dúvidas e angústias expressam-se em forma de palavras, frases e discursos nas multivariadas situações de saúde e doença. Especificamente, as unidades de registros produzidas pelos enfermeiros da APS convidam, com base nos termos de ordem "barreira linguística", "idioma" e "língua", à discussão da comunicação verbal prejudicada pela diversidade cultural ocasionada pelo fluxo migratório venezuelano.

Isso fica evidenciado pela rápida articulação de palavras na fala, necessidade de repetição de mensagens, esforço para compreender os núcleos de sentido das informações dialogadas, choque idiomático português-espanhol e incompreensão parcial ou total do que é dito verbalmente nos encontros de cuidado estabelecidos entre o enfermeiro da APS e o migrante venezuelano.

\section{Discussão}

Trazer à tona reflexões sobre a mistura de dois idiomas distintos presentes nas cenas de cuidar envolvendo enfermeiros e migrantes venezuelanos no âmbito da APS é desafiador. As discussões possuem variadas possibilidades de entrada, sobretudo quando a migração venezuelana é discutida à luz da subjetividade, isto é, atravessada por instabilidades políticas, conflitos que ameaçam a segurança pública dos Estados Nacionais, racismo e xenofobia, exclusão social, fatores de risco ligados às condições de trabalho e dificuldade para acessar os serviços de saúde ${ }^{(12)}$.

Isso porque o ser migrante "[...] tudo carrega em seu corpo; carrega seus pertences, carrega física e/ou emocionalmente seus familiares e filhos; carrega também seus contatos, seus sonhos; carrega seu próprio corpo e em seu próprio corpo, as marcas de sua história. Além disso, o corpo que migra ocupa espaço, vê, observa e é visto e observado; julga e é julgado, é aceito ou rejeitado, muitas vezes devido à sua aparência física, porque tudo e cada parte do corpo está marcada, por vezes estigmatizada culturalmente" ${ }^{\text {(13:9) }}$.

As adversidades enfrentadas nos planos social, econômico, jurídico, político e cultural pelos migrantes venezuelanos não são poucas. Os indivíduos e os grupos em (trans)formação aprendem a lidar com a alteridade em diferentes frentes: legislação, costumes, crenças, preconceitos, conceitos e idioma ${ }^{(14)}$.

Para ilustrar essa afirmação, a chegada de milhares de venezuelanos em Boa Vista vem modificando o cenário local. Ao percorrer a cidade, é possível identificar várias fachadas e estabelecimentos comerciais contendo um conjunto fragmentado de mensagens com nomes e termos em espanhol, numa clara disputa do idioma espanhol com o português ${ }^{(15)}$. Não saber o idioma representa um elemento complicador para integração desse grupo social. Em sua grande maioria, os migrantes venezuelanos não dominam o português e não falam outra língua que não o espanhol. Em parte, essa dificuldade linguística é amenizada pela rede migratória que muitos deles afirmam possuir no pais ${ }^{(16)}$.

Toda esta forma de construção da migração venezuelana aponta para uma necessidade urgente de expansão da resposta de saúde humani tária com o objetivo de garantir acesso dos venezuelanos aos serviços de saúde e a proteção da saúde pública nas áreas de fronteira. No Brasil, os migrantes venezuelanos enfrentam dificuldades de acesso aos serviços, devido às acentuadas barreiras linguísticas ${ }^{(17)}$.

A barreira da língua dificulta o estabelecimento de uma boa comunicação, o que afeta a relação usuário migrante-profissional de saúde e a adesão aos tratamentos, bem como o bom controle do processo assistencial. As barreiras de comunicação podem apresentar-se de diversas formas, entretanto, especificamente na relação enfermeiro-paciente, são identificadas principalmente a linguagem técnica, o idioma, a cultura e a falta de empatia ${ }^{(16)}$. 
O idioma como indutor de comunicação verbal prejudicada é considerado uma barreira. Esta é intensificada "[...] quando a doença é mais grave, devido à maior utilização pelos profissionais de termos técnicos para esclarecê-la, além da dificuldade de compreensão das instruções durante os procedimentos que dependem da colaboração dos pacientes para serem realizados"(18:126).

Em vista disso, é importante pensar que o idioma é considerado um importante elemento de barreira assistencial, com base no qual vínculos assistenciais podem ser produzidos. Além disso, a resposta do usuário migrante frente ao plano terapêutico pode ser efetivamente avaliada. Quando o enfermeiro recusa-se ou não está inclinado a adaptar-se às barreiras linguísticas no cuidado prestado, uma das principais estratégias para a promoção da saúde e prevenção de agravos, que é a educação em saúde, fica seriamente comprometida. Isso fragiliza não só o cuidado de enfermagem, mas também a assistência global em saúde ${ }^{(2)}$.

A produção do cuidado de enfermagem, representado pelos encontros estabelecidos entre os enfermeiros e os migrantes venezuelanos na APS, é carregada de expectativas a priori e de interferências mútuas, que dão um caráter de imprevisibilidade ao produto do trabalho em saúde; como o encontro acontece em ato, ele é parcialmente incontrolável $^{(4)}$.

Essas interferências são representadas por domínios da linguagem, idiomas e dialetos, que, no campo do cuidado em saúde, (re)constroem caminhos articulados com os migrantes venezuelanos, principalmente quando os enfermeiros da APS solicitam-lhes que falem devagar, esperam que se acalmem para falar, repetem exaustivamente orientações em saúde e se esforçam para compreender a língua espanhola.

Em razão dos problemas linguísticos que podem tornar a comunicação verbal prejudicada, é importante, no relacionamento interpessoal junto aos processos de cuidado implementados na APS, que "[...] os profissionais de saúde tenham sensibilidade para compreender o teor das mensagens enviadas pelos pacientes, de forma a prestar uma assistência mais eficiente e adequada às suas necessidades"(18:126).

Dentre as estratégias de enfermagem adotadas para ultrapassar as barreiras culturais e linguísticas, encontram-se o atendimento às necessidades emocionais e psicológicas da pessoa culturalmente diversa. Além disso, conflitos culturais são evitados e adaptações dos discursos à linguagem são implementados ${ }^{(19)}$.

Para isso, é necessário que o enfermeiro tenha competência cultural e política, compreenda os valores, as crenças, os rituais e o modo de vida dos migrantes venezuelanos e das suas famílias, numa perspectiva de construção de um novo paradigma do cuidar. Este deve integrar não só uma abordagem culturalmente competente, como também deve visar a promoção da saúde, a qualidade de vida e o bem-estar, mediante o uso de recursos linguísticos reconhecidos dos enfermeiros da APS, tais como a presença de um acompanhante capaz de traduzir a língua e a existência de tradutores da respectiva instituição ${ }^{(20-21)}$. Além da proposta de inclusão de intérpretes nos serviços primários de saúde, considera-se ainda a opção de aperfeiçoamento da equipe, mediante o aprendizado da língua espanhola, bem como de aspectos culturais do povo venezuelano que possam interferir diretamente na saúde desses indivíduos.

Não é razoável exigir que os venezuelanos que passam pelo inesperado processo de migração forçada sejam penalizados, em termos assistenciais em saúde, por ainda não dominarem o idioma do país acolhedor. Pelo contrário, os enfermeiros são cotidianamente convidados a redobrarem os esforços para atingir uma comunicação eficiente, ou mesmo cobrar dos serviços de saúde capacitações para lidar com essa problemática da comunicação verbal prejudicada, considerando os fluxos migratórios locorregionais ${ }^{(2)}$.

A comunicação verbal prejudicada é concebida como diagnóstico presente na classificação taxonômica da NANDA I (2018-2020) e definida como a "[...] capacidade diminuída, retardada ou ausente para receber, processar, transmitir e/ou usar um sistema de símbolos"(22:503). No plano das 
migrações, pistas qualitativas de sua existência foram evidenciadas nos depoimentos dos enfermeiros da APS, ao registrarem dificuldades para falar, formar frases, formar palavras e verbalizar.

Esta discussão não se restringe aos Diagnósticos de Enfermagem da NANDA- ${ }^{(22)}$. Considera-se necessário pensar a comunicação verbal ineficaz entre o migrante venezuelano e o profissional da saúde no campo interprofissional, em que a linguagem oficial é a Classificação Internacional de Atenção Primária (CIAP) ${ }^{(23)}$. Nesse sentido, a identificação dos motivos de consulta expressados pelo usuário migrante, seu problema de saúde ou procedimento realizado, promove um registro coerente e uniformizado pelo enfermeiro e, por conseguinte, melhor comparação de informações entre as unidades de saúde.

Justamente pela complexidade da dimensão comunicativa, parece necessária a consideração desse diagnóstico de enfermagem nos instrumentos de registro e consolidação de dados de atendimento do e-SUS APS, sobretudo os que utilizam a CIAP em regiões de fronteira, onde os fluxos migratórios ocorrem com maior intensidade. Trata-se de um diagnóstico utilizado pelo enfermeiro na prática clínico-assistencial ainda de maneira tímida, considerando aspectos principalmente físicos, de fácil identificação, sem avaliações precisas e detalhadas do prejuízo na comunicação verbal $^{(9)}$.

É fundamental pensar que o enfermeiro da APS, na interrelação com o migrante venezuelano, precisa estar vigilante quanto ao uso adequado das técnicas da comunicação interpessoal. Somente pela comunicação efetiva é que ele poderá ajudar o paciente a conceituar seus problemas, enfrentá-los, visualizar sua participação na experiência e identificar alternativas de solução, além de auxiliá-lo a encontrar novos padrões de comportamento ${ }^{(24)}$.

Nesse sentido, é necessário, igualmente, refletir sobre o não dito verbalmente pelo corpo do migrante venezuelano, isto é, sobre a comunicação não verbal como essencial para validação da fala. Sabe-se que a APS é um campo muito amplo para o enfermeiro, sendo necessário o domínio de várias competências para realizar seu trabalho com eficácia, dentre as quais destacam-se: "[...] gerenciamento do seu processo de trabalho, raciocínio clínico e abstrato, planejamento, comunicação, administração do tempo, conhecimento técnico-científico da área [...]",(25:755) e comunicação, o que inclui a dimensão verbal e não verbal ${ }^{(25)}$.

Elencam-se, a esse raciocínio, envolvendo o processo comunicacional, angulações teóricas de natureza antropológica e inclinações práticas (trans)culturais para o interior dos cuidados desenvolvidos pelo enfermeiro junto ao migrante venezuelano na APS. Assim, os modos de comunicação e a linguagem descobertos nos cuidados precisam ser incorporados no ambiente social em que o trabalho e as pesquisas em saúde são produzidos.

Considera-se como limitação deste estudo a redução do grupo social, ocorrida pela inviabilidade de produção de dados em todas as UBS, motivada pela pandemia da doença Coronavírus. Nesse sentido, é reconhecida a necessidade de ampliação do número de UBS, bem como a consideração, nas futuras investigações, dos migrantes haitianos e guianenses. Isso porque, acredita-se que as matrizes culturais e linguísticas desses povos criam singulares relevos assistenciais no campo da APS. Pondera-se ainda, como elemento limitador, a sua natureza analítica ser centrada em unidades conteudistas que versam especificamente sobre a comunicação verbal. Há que se considerar, em estudos futuros, a dimensão expressiva, aqui representada por elementos da comunicação não verbal presentes no cuidado produzido pelo enfermeiro junto aos migrantes venezuelanos.

No que tange às contribuições para a área da enfermagem, o estudo fornece reflexões no campo qualitativo sobre as estratégias e dificuldades que os enfermeiros apresentam para implementar seus cuidados junto aos migrantes venezuelanos na APS. Acredita-se que aqueles atuantes no extremo Norte do Brasil e que desenvolvem atividades assistenciais em UBS localizadas em regiões de fronteira possam utilizar as discussões nos processos educacionais e gerenciais, como forma de subsidiar a melhoria da 
qualidade da comunicação verbal e, consequentemente, a efetivação de ações de cuidar seguras e resolutivas aos migrantes.

\section{Considerações Finais}

Pode-se dizer que este estudo está alicerçado nas dimensões existenciais envolvidas no processo de migração venezuelana para o Brasil, que se desvela com base no que é comunicado verbalmente. As produções de reflexões deixam pistas sinalizadoras de que a comunicação verbal, realizada por enfermeiros da APS no cuidado voltado ao migrante venezuelano, é atravessada por elementos linguísticos.

O idioma foi considerado um elemento indutor de comunicação verbal prejudicada no cuidado ocorrido na APS. A barreira linguística foi evidenciada quando os enfermeiros, no interior das práticas de cuidar, apontaram criar estratégias para emitirem e receberem mensagens junto aos migrantes venezuelanos. O falar pausadamente, a repetição de mensagens e o esforço para compreensão da língua espanhola representaram os esforços dos enfermeiros para cuidar do migrante venezuelano em uma matriz cultural diferenciada.

Nesse prisma, as reflexões postas abrem possibilidades investigativas para os enfermeiros pensarem a comunicação verbal prejudicada no plano (trans)cultural. Um objeto investigativo a ser explorado com outras vertentes metodológicas e referenciais teóricos da antropologia, com vistas a ampliar o que se sabe sobre os povos migrantes venezuelanos localizados na Amazônia Legal.

Cabe ainda ponderar que, no percurso investigativo, foi descoberta a necessidade de apostas científicas que envolvessem elementos expressivos do corpo migrante venezuelano, isto é, tornar-se fundamental a continuidade de estudos que considerem o regime dos signos, da expressão corporal e do que é produzido como comunicação não verbal no campo da APS.

Ressalta-se ainda o convite para gestores de saúde pensarem em projetos ou programas de atenção à saúde direcionados para enfermeiros e população migrante venezuelana. Fala-se de microespaços de acolhimento, que apresentam intérpretes para possibilitar a compreensão completa do que está sendo emitido e recebido como mensagem de cuidar.

Assim, com a certeza do inacabado, acredita-se que as reflexões produzidas pelos discursos dos enfermeiros da RAPS de Boa Vista (RR), que dizem respeito à comunicação verbal, no encontro com migrantes venezuelanos, possam contribuir com os processos gerenciais, assistenciais, educacionais e para o fortalecimento dos princípios e diretrizes do Sistema Único de Saúde, beneficiando o desenvolvimento de práticas (trans)culturais capazes de considerar as especificidades migratórias em regiões de fronteira.

\section{Contribuições:}

O autor é responsável pela concepção do projeto, análise e interpretação dos dados, redação do artigo, revisão e aprovação final da versão a ser publicada, e por todos os aspectos do trabalho, na garantia da exatidão e integridade de qualquer parte da obra.

\section{Referências}

1. Abubakar I, Aldridge RW, Devakumar D, Orcutt M, Burns R, Barreto ML, et al. The UCL-Lancet Commission on Migration and Health: the health of a world on the move. Lancet. 2018;392(15):2606-54. DOI: 10.1016/S01406736(18)32114-7

2. Arruda-Barbosa L, Sales AFG, Torres ME. Impacto da migração venezuelana na rotina de um hospital de referência em Roraima, Brasil. Interface (Botucatu). 2020;24:e190807. DOI: 10.1590/Interface.190807

3. Souza AR, Silveira MCP. O fluxo migratório de venezuelanos para o Brasil (2014-2018): análise do arcabouço jurídico brasileiro e da conjuntura interna venezuelana. Cad Prolam/USP. 2018;17(32):114-32. DOI: 10.11606/issn.1676-6288. prolam.2018.144270

4. Merhy EE, Feuerwerker LCM, Santos MLM, Bertussi DC, Baduy RS. Rede Básica, campo de forças e micropolítica: implicações para a gestão 
e cuidado em saúde. Saúde Debate. 2019;43 (Esp. 6):70-83. DOI: 10.1590/0103-11042019S606

5. Silva PS, Arruda-Barbosa L. Imigração de venezuelanos e os desafios enfrentados por enfermeiros da Atenção Primária à Saúde. Enferm Foco [Internet]. 2020 [cited 2021 Jun 10];11(2):37-43. Available from: http://revista. cofen.gov.br/index.php/enfermagem/article/view/ 3091/768

6. Veliz-Rojas L, Bianchetti-Saavedra AF, SilvaFernández M. Competencias interculturales en la atención primaria de salud: un desafío para la educación superior frente a contextos de diversidad cultural. Cad Saúde Pública. 2019;35(1):e00120818. DOI: 10.1590/0102-311X00 120818

7. Araújo MMT, Silva MJP. Estratégias de comunicação utilizadas por profissionais de saúde na atenção à pacientes sob cuidados paliativos. Rev Esc Enferm USP. 2012;46(3):626-32 DOI: $10.1590 /$ S0080-62342012000300014

8. Barbosa IA, Silva MJP. Cuidado de enfermagem por telessaúde: qual a influência da distância na comunicação? Rev Bras Enferm. 2017;70(5)928-34. DOI: 10.1590/0034-7167-2016-0142

9. Puggina ACG, Trovo MM, Biondo CA, Barbosa IA, Santos M, Silva MJP. Diagnóstico de enfermagem comunicação verbal prejudicada na prática clínica: uma revisão integrativa. REFACS (online). 2016;4(2):135-44. DOI: 10.18554/refacs.v4i2.1644

10. Minayo MCS. Origem inusitada da pesquisa qualitativa em ciências sociais no Brasil. Hist Ciênc Saúde - Manguinhos. 2020;27(3):919-32. DOI: $10.1590 /$ s0104-59702020000400012

11. Bardin L. L'analyse de contenu. Paris: Presses Universitaires de France; 2013.

12. Ordóñez JT, Ramírez Arcos HE. (Des)orden nacional: la construcción de la migración venezolana como una amenaza de salud y seguridad pública en Colombia. Rev Cienc Salud. 2019;17(Esp.):48-68. DOI: 10.12804/revistas. urosario.edu.co/revsalud/a.8119

13. Cavalcanti L, Ennes MA, Oliveira M. Editorial Migrações e Circularidade: Teoria, Políticas de Estado e Realidades Migrantes. Rev Bras Sociol. 2020;8(19):7-25. DOI: 10.20336/rbs.761

14. Santos SMA, Meza IJB. Para onde vou com a minha família? Uma etnografia sobre projetos coletivos e migração venezuelana em Manaus (Brasil). Rev
Interdiscip Mobil Hum. 2021;29(61):179-94. DOI: $10.1590 / 1980-85852503880006111$

15. Morais VMI, Lima DM. O horizonte comunicativo da migração venezuelana na cidade de Boa Vista - Roraima. Dossiê. Textos Debates [Internet]. 2019 [cited 2021 Jun 10];(32):199-212. Available from: https://revista.ufrr.br/textosedebates/article/ view/5698/pdf

16. Estevan MDG, Solano Ruíz MC. La aplicación del modelo de competencia cultural en la experiencia del cuidado en profesionales de Enfermería de Atención Primaria. Aten Primaria. 2017;49(9):549-56. DOI: 10.1016/j.aprim.2016.10.013

17. Doocy S, Page KR, Hoz F, Spigel P, Beyer C. Venezuelan Migration and the Border Health Crisis in Colombia and Brazil. J Migr Hum Secur. 2019;7(3):79-91. DOI: 10.1177/2331502419860138

18. Guerra K, Ventura M. Bioética, imigração e assistência à saúde: tensões e convergências sobre o direito humano à saúde no Brasil na integração regional dos países. Cad saúde colet. 2017;25(1):123-9. DOI: 10.1590/1414-462x2017000 10185

19. Gaspar AM, Branco CB, Pedro CF, Nunes DF, Alves NS, Reis A. As estratégias de enfermagem adotadas para ultrapassar as barreiras culturais e linguísticas com pessoas culturalmente diversas uma scoping review. Rev UIIPS. 2020;8(1):215-22. DOI: 10.25746/ruiips.v8.i1.19893

20. Rezende LC, Caram CS, Caçador BS, Brito MJM. Prática do enfermeiro em comunidades quilombolas: interface entre competência cultural e política. Rev Bras Enferm. 2020;73(5):e20190433. DOI: 10.1590/0034-7167-2019-0433

21. Coutinho E, Amaral S, Parreira V, Chaves C, Amaral O, Nelas P. O cuidado cultural na trajetória da enfermagem transcultural e competência cultural. CIAIQ - Investigação Qualitativa em Saúde [Internet]. 2017 [cited 2021 Jun 10];2:157887. Available from: https://proceedings.ciaiq.org/ index.php/ciaiq2017/article/view/1510/1467

22. Herdman ST, Kamitsuru S. Diagnósticos de enfermagem da NANDA I: definições e classificação, 2018-2020. 11a ed. Porto Alegre: Artmed; 2018.

23. Sociedade Brasileira de Medicina de Família e Comunidade. Classificação Internacional de Atenção Primária (CIAP 2). Florianópolis: Armazém Digital Editoração Eletrônica; 2010. 
24. Silva MJP. Comunicação tem remédio: a comunicação nas relações interpessoais em saúde. 8a ed. São Paulo: Loyola; 2011.

25. Ferreira SRS, Périco LAD, Dias VRFG. A complexidade do trabalho do enfermeiro na Atenção Primária à Saúde. Rev Bras Enferm.
2018;71(Supl 1):704-9. DOI: 10.1590/0034-71672017-0471

Recebido: 9 de julho de 2021 Aprovado: 17 de setembro de 2021 Publicado: 4 de novembro de 2021

A Revista Baiana de Enfermagem utiliza a Licença Creative Commons - Atribuição-NãoComercial 4.0 Internacional. https://creativecommons.org/licenses/by-nc/4.0/ Este artigo é de acesso aberto distribuído sob os termos da Licença Creative Commons (CC BY-NC). Esta licença permite que outros remixem, adaptem e criem a partir do seu trabalho para fins não comerciais. Embora os novos trabalhos tenham de lhe atribuir o devido crédito e não possam ser usados para fins comerciais, os usuários não têm de licenciar esses trabalhos derivados sob os mesmos termos. 